\title{
Being Short, Sweet, and Sour: Congruent Visuo-Olfactory Stimulation Enhances Illusory Embodiment
}

\author{
Roel Lesur, Marte ; Aicher, Helena ; Delplanque, Sylvain ; Lenggenhager, Bigna
}

\begin{abstract}
Bodily self-identification has shown to be easily altered through spatiotemporally congruent multimodal signals. While such manipulations are mostly studied through visuo-tactile or visuo-motor stimulation, here we investigated whether congruent visuo-olfactory cues might enhance illusory selfidentification with an arbitrary object. Using virtual reality, healthy individuals saw a grapefruit from its supposed first-person perspective that was touched in synchrony with their own body. The touch attempted to replicate what was seen as softly squeezing the grapefruit. Crucially, when we additionally presented the smell of a grapefruit in synchrony with the squeezing, they self-identified stronger with the fruit than when they smelled strawberry.
\end{abstract}

DOI: https://doi.org/10.1177/0301006620928669

Posted at the Zurich Open Repository and Archive, University of Zurich

ZORA URL: https://doi.org/10.5167/uzh-194495

Journal Article

Accepted Version

Originally published at:

Roel Lesur, Marte; Aicher, Helena; Delplanque, Sylvain; Lenggenhager, Bigna (2020). Being Short, Sweet, and Sour: Congruent Visuo-Olfactory Stimulation Enhances Illusory Embodiment. Perception, 49(6):693-696.

DOI: https://doi.org/10.1177/0301006620928669 
CONGRUENT VISUO-OLFACTORY CUES ENHANCE EMBODIMENT

Being short, sweet and sour: congruent visuo-olfactory stimulation enhances illusory embodiment

Marte Roel Lesur ${ }^{1,2}$, Helena Aicher ${ }^{1}$, Sylvain Delplanque ${ }^{3}$, Bigna Lenggenhager ${ }^{1}$

${ }^{1}$ Department of Psychology, University of Zurich, Zurich, Switzerland

${ }^{2}$ BeAnotherLab

${ }^{3}$ Swiss Center for Affective Sciences, University of Geneva-CISA, Geneva, Switzerland

Corresponding Author :

Marte Roel Lesur, University of Zurich, Department of Psychology, Binzmühlestrasse 14, Box 9, 8050, Zurich, Switzerland. Email: marteroel@gmail.com.

Keywords :

Body perception, Multisensory/cross-modal processing, Spatiotemporal factors, Smell, Proprioception 


\begin{abstract}
:
Bodily self identification has shown to be easily altered through spatiotemporally congruent multimodal signals. While such manipulations are mostly studied through visuo-tactile or visuomotor stimulation, here we investigated whether congruent visuo-olfactory cues might enhance illusory self-identification with an arbitrary object. Using virtual reality, healthy individuals saw a grapefruit from its supposed first-person perspective that was touched in synchrony with their own body. The touch attempted to replicate what was seen as softly squeezing the grapefruit. Crucially, when we additionally presented the smell of a grapefruit in synchrony with the squeezing, they self-identified stronger with the fruit than when they smelled strawberry.
\end{abstract}


CONGRUENT VISUO-OLFACTORY CUES ENHANCE EMBODIMENT

\section{Being short, sweet and sour: congruent visuo-olfactory stimulation enhances illusory embodiment}

The sense of body has been shown to be remarkably plastic, to the point that healthy participants can feel illusory embodiment over notably different bodies than their own (e.g. Petkova \& Ehrsson, 2008; Hoort, Guterstam, \& Ehrsson, 2011; Ahn et al., 2016), when there is spatiotemporal congruence between various senses. Such newly established multisensory contingencies are assumed to temporarily overrule longer-term priors about the body (Apps \& Tsakiris, 2014). In these examples, the visually shown body still roughly maps to the human body metrics, however the embodiment of more arbitrary objects is still hardly investigated and subject to debate (Kalckert et al., 2019; Tsakiris et al., 2010). Previous studies have mostly used senses that can easily be mapped spatially, such as vision, touch and proprioception to induce illusory embodiment. Other, less primarily-spatial senses like olfaction have largely been neglected in the literature (see Brianza et al., 2019, for an exception). Compared to the strength of other bodily contingencies (e.g. visuo-tactile), scents may be a mild cue to elicit illusory embodiment. Yet, we speculate that scent would be more important when embodying an arbitrary non-humanoid object, especially when it has a strong prior association with an odor.

We designed an immersive experience in which participants saw on a head mounted display (HMD; Oculus Rift CV1, Oculus, USA) a 235-degree video recorded from the upper part of a grapefruit (see Figure 1A). Head-movements were tracked (using the HMD's internal tracking system and an Oculus Sensor) and matched to the optic flow enabling a natural and free exploration of the environment, which has been proposed to be an important modulator of embodiment, especially in situations of temporal mismatches of other bodily signals (Roel Lesur et al., 2018). The seen fruit was repetitively squeezed (approximately every $5 \mathrm{~s}$ ) while the 


\section{CONGRUENT VISUO-OLFACTORY CUES ENHANCE EMBODIMENT}

participants' shoulders were simultaneously pressed by the experimenter (visuo-tactile congruency) from the front. To achieve synchrony, the timing was practiced beforehand and the video monitored in real time. Simultaneous to the squeezing an olfactory pen with either the synthesized scent of a grapefruit (visuo-olfactory congruency) or of a strawberry (visuo-olfactory incongruency) was held below the participant's nose. The video was prerecorded placing a 235degree camera (SP 360 4K, Kodak, USA) on top of the grapefruit, as if from a "first person perspective", which was then mapped to a mesh matching the distortion of the camera lens using software developed Unity (2018.3.4; Unity Technologies, USA) and played back on the HMD.

Data were recorded within a science exhibition in a museum (Technorama, Switzerland), 21 participants took part in the experiment. They were explained about the virtual reality experience and verbally agreed to participate before putting on and calibrating the HMD. They were allowed to move their head. The order of the conditions (grapefruit/strawberry scent) was counterbalanced between participants. After each condition we measured self-identification with the grapefruit using a single question (See Figure 1B) to be answered on a Likert scale ranging from 1-7, respectively corresponding to not at all and completely. A two-tailed Wilcoxon signed rank test revealed a significantly higher score in the congruent compared to the incongruent conditions $(\mathrm{p}=0.003, \mathrm{~W}=124, \mathrm{r}=0.46)$ (Figure $1 \mathrm{~B})$. A posterior test with 10 naïve blindfolded participants confirmed that the odors were distinguishable $(90 \%$ recognized the grapefruit as a citrus and $80 \%$ the strawberry as such). 

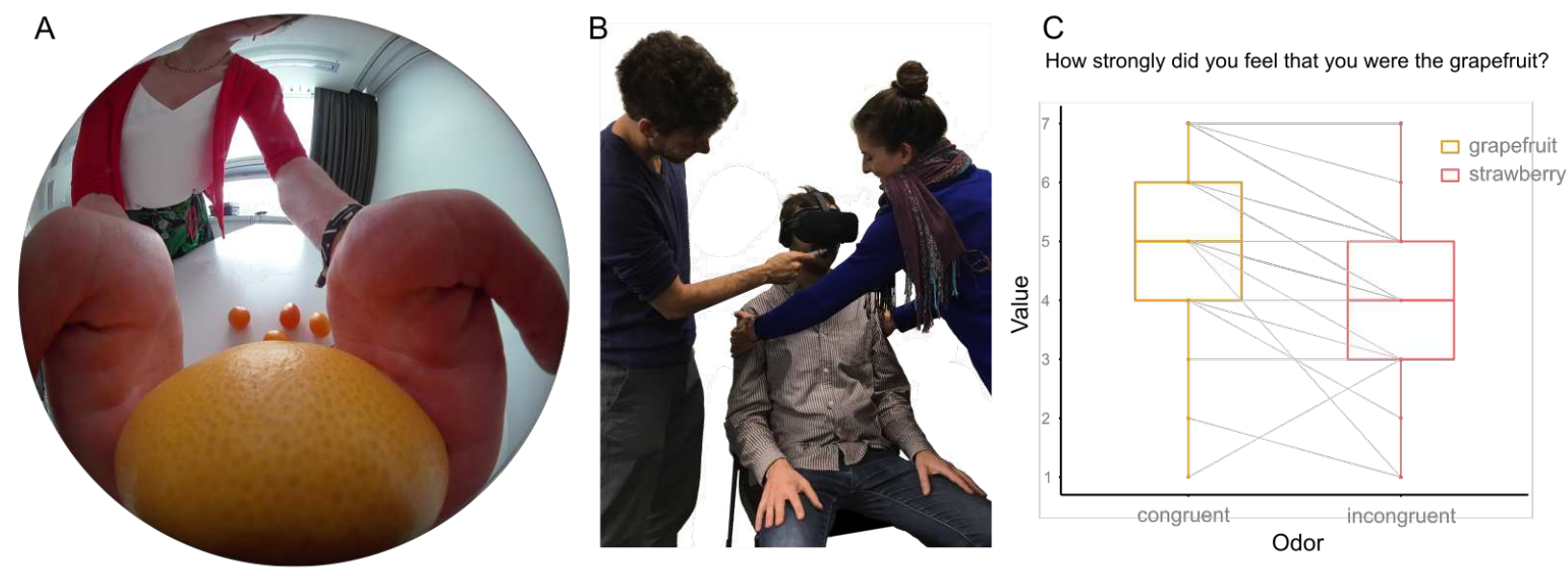

Figure 1. A) Single frame of the video, showing the 'perspective' of the grapefruit being squeezed, B) corresponding tactile and olfactory stimulation C) Medians, IQR, and participants' responses connected by lines between conditions for the question addressing self-identification with the grapefruit.

We consider these results as preliminary given the public experimental setting and the single explicit measure applied however they point at associations between scent and objects potentially playing a role during illusory ownership illusions. This may be relevant to further understand the multimodal nature of the sense of body, as olfaction has been an often-neglected modality in the field. While crossmodal associations between odor and other senses and abstract concepts have been found (Hanson-Vaux et al., 2013; Seo et al., 2010), olfaction may have further impact in multimodal perception given its apparent contribution to illusory embodiment. Future studies should address important issues such as whether the change in the presented odor was consciously perceived as olfaction has been suggested to often escape conscious awareness (Köster, 2002); and how much the salience of the mismatch affects embodiment. Objective measures are further encouraged, such as skin conductance responses to threat, which have previously been associated to illusory embodiment (Petkova \& Ehrsson, 2008). Furthermore, to 
CONGRUENT VISUO-OLFACTORY CUES ENHANCE EMBODIMENT

further test our initial speculations, various objects with different degree of humanoid shapes and different olfactory association strengths could be tested. Odor and the sense of body have often been elusive for experimental research for practical and theoretical reasons, yet this preliminary study may open experimental possibilities for furthering our knowledge in the field.

\section{References}

Apps, M. A. J., \& Tsakiris, M. (2014). The free-energy self: A predictive coding account of selfrecognition. Neuroscience \& Biobehavioral Reviews, 41, 85-97. https://doi.org/10.1016/j.neubiorev.2013.01.029

Brianza, G., Tajadura-Jiménez, A., Maggioni, E., Pittera, D., Bianchi-Berthouze, N., \& Obrist, M. (2019). As Light as Your Scent: Effects of Smell and Sound on Body Image Perception. In D. Lamas, F. Loizides, L. Nacke, H. Petrie, M. Winckler, \& P. Zaphiris (Eds.), Human-Computer Interaction - INTERACT 2019 (Vol. 11749, pp. 179-202). Springer International Publishing. https://doi.org/10.1007/978-3-030-29390-1_10

Hanson-Vaux, G., Crisinel, A.-S., \& Spence, C. (2013). Smelling Shapes: Crossmodal Correspondences Between Odors and Shapes. Chemical Senses, 38(2), 161-166. https://doi.org/10.1093/chemse/bjs087

Kalckert, A., Bico, I., \& Fong, J. X. (2019). Illusions With Hands, but Not With BalloonsComparing Ownership and Referral of Touch for a Corporal and Noncorporal Object After Visuotactile Stimulation. Perception, 48(5), 447-455. https://doi.org/10.1177/0301006619839286

Köster, E. P. (2002, October). The Specific Characteristics of the Sense of Smell. Olfaction, Taste, and Cognition. https://doi.org/10.1017/CBO9780511546389.007 
CONGRUENT VISUO-OLFACTORY CUES ENHANCE EMBODIMENT

Petkova, V. I., \& Ehrsson, H. H. (2008). If I Were You: Perceptual Illusion of Body Swapping. PLOS ONE, 3(12), e3832. https://doi.org/10.1371/journal.pone.0003832

Roel Lesur, M., Gaebler, M., Bertrand, P., \& Lenggenhager, B. (2018). The Plasticity of the Bodily Self: Head Movements in Bodily Illusions and Their Relation to Gallagher's Body Image and Body Schema. Constructivist Foundations, 14(1), 94-105.

Seo, H.-S., Arshamian, A., Schemmer, K., Scheer, I., Sander, T., Ritter, G., \& Hummel, T. (2010). Cross-modal integration between odors and abstract symbols. Neuroscience Letters, 478(3), 175-178. https://doi.org/10.1016/j.neulet.2010.05.011

Tsakiris, M., Carpenter, L., James, D., \& Fotopoulou, A. (2010). Hands only illusion: Multisensory integration elicits sense of ownership for body parts but not for noncorporeal objects. Experimental Brain Research, 204(3), 343-352. https://doi.org/10.1007/s00221-009-2039-3

\section{Acknowledgement}

This project was funded by the UNIGE - UZH Joint Seed Money Funding 2018. MRL and BL were funded by the Swiss National Science Foundation (PP00P1_170511) and HA by the Neuroscience Center Zurich (ZNZ PhD Grant 2018). We thank the Swiss Science Center Technorama, especially Armin Duff, for enabling data collection. 